\title{
Vanilloid Receptors Presynaptically Modulate Cranial Visceral Afferent Synaptic Transmission in Nucleus Tractus Solitarius
}

\author{
Mark W. Doyle, Timothy W. Bailey, Young-Ho Jin, and Michael C. Andresen \\ Department of Physiology and Pharmacology, Oregon Health and Science University, Portland, Oregon 97201-3098
}

Although the central terminals of cranial visceral afferents express vanilloid receptor 1 (VR1), little is known about their functional properties at this first synapse within the nucleus tractus solitarius (NTS). Here, we examined whether VR1 modulates afferent synaptic transmission. In horizontal brainstem slices, solitary tract (ST) activation evoked EPSCs. Monosynaptic EPSCs had low synaptic jitter (SD of latency to successive shocks) averaging $84.03 \pm 3.74 \mu \mathrm{sec}(n=72)$ and were completely blocked by the non-NMDA antagonist 2,3dihydroxy-6-nitro-7-sulfonyl-benzo[f]quinoxaline (NBQX). Sustained exposure to the VR1 agonist capsaicin (CAP; $100 \mathrm{~nm}$ ) blocked ST EPSCs (CAP-sensitive) in some neurons but not others (CAP-resistant). CAP-sensitive EPSCs had longer latencies than CAP-resistant EPSCs $(4.65 \pm 0.27 \mathrm{msec}, n=48$ vs $3.53 \pm 0.28$ msec, $n=24$, respectively; $p=0.011$ ), but they had similar jitter. CAP evoked two transient responses in CAPsensitive neurons: a rapidly developing inward current $\left(I_{\text {cap }}\right)$
$(108.1 \pm 22.9 \mathrm{pA} ; n=21)$ and an increase in spontaneous synaptic activity. After 3-5 min in CAP, $I_{\text {cap }}$ subsided and ST EPSCs disappeared. NBQX completely blocked $I_{\text {cap. The VR1 }}$ antagonist capsazepine (10-20 $\mu \mathrm{M})$ attenuated CAP responses. Anatomically, second-order NTS neurons were identified by 4-(4dihexadecylamino)styryl)- $N$-methylpyridinium iodide transported from the cervical aortic depressor nerve (ADN) to stain central terminals. Neurons with fluorescent ADN contacts had CAPsensitive EPSCs $(n=5)$ with latencies and jitter similar to those of unlabeled monosynaptic neurons. Thus, consistent with presynaptic VR1 localization, CAP selectively activates a subset of ST axons to release glutamate that acts on non-NMDA receptors. Because the CAP sensitivity of cranial afferents is exclusively associated with unmyelinated axons, VR1 identifies C-fiber afferent pathways within the brainstem.

Key words: sensory; vanilloid; glutamate; presynaptic modulation; autonomic; visceral; baroreceptor; baroreflex
Many afferents from visceral organs send information into the CNS through the cranial nerves to terminate in the brainstem at the nucleus tractus solitarius (NTS) (Loewy, 1990). These cranial visceral afferents are often considered to be separate and distinct from the spinal sensory neurons of the dorsal root ganglia because of their very different physiological functions (Saper, 2000). Cranial visceral afferents contribute to autonomic, hormonal, and homeostatic central regulatory mechanisms, all of which are generally unconscious. These cranial visceral afferents fall into two general classes, myelinated and unmyelinated, and share a number of general cellular and molecular characteristics with spinal sensory neurons (Lawson, 1992). However, much less is known about the central processing within the pathways of cranial visceral afferents compared with their somatic afferent counterparts.

Although vanilloid receptor 1 (VR1) is strongly linked to spinal nociceptive neurons (Caterina and Julius, 2001), immunohistochemical and in situ hybridization data indicate that VR1 is present in cranial sensory neurons (Burnstock, 2000). VR1 is expressed at a subset of cranial visceral afferent cell bodies in the nodose ganglion (Helliwell et al., 1998; Tominaga et al., 1998; Mezey et al., 2000). These cranial afferents enter the brainstem to form the solitary tract (ST) before terminating in the NTS

\footnotetext{
Received April 5, 2002; revised June 10, 2002; accepted June 13, 2002.

This work was supported by National Institutes of Health Grant HL-41119 and grants from the National Center of the American Heart Association.

Correspondence should be addressed to Dr. Michael C. Andresen, Department of Physiology and Pharmacology, Oregon Health and Science University, Portland, Oregon 97201-3098. E-mail: andresen@ohsu.edu.

M. W. Doyle's present address: Department of Biology, George Fox University, Newberg, OR 97132-2697.

Copyright (C) 2002 Society for Neuroscience $\quad 0270-6474 / 02 / 228222-08 \$ 15.00 / 0$
}

(Loewy, 1990); VR1 is found within these central terminal fields (Guo et al., 1999). This visceral primary afferent synapse is likely an important point of modulation in the integration of major autonomic reflexes such as the baroreceptor reflex (Andresen et al., 2001).

Here, we examined whether VR1 activation interacts with synaptic transmission between cranial visceral primary afferents and second-order neurons within the NTS. We electrophysiologically identified second-order neurons using measures of the synaptic reliability of EPSCs. Our horizontal brainstem slice is optimized to isolate the electrical activation of cranial visceral afferents during ST stimulation from potential contamination by the activation of local interneurons (Miles, 1986; Andresen and Yang, 1990). In addition, we identified second-order NTS neurons by the presence of fluorescent terminals from aortic baroreceptors (Mendelowitz et al., 1992). Our results suggest that capsaicin (CAP) and the presence of VR1 receptors on ST processes identify two afferent pathways (CAP-sensitive and CAPresistant) that are presynaptically distinct. Together with the study by Bailey et al. (2002) that suggests postsynaptic differentiation of these NTS neurons in their action potential transmission characteristics, this CAP work reveals that the differentiation of reflex pathways through the brainstem begins at the secondorder neurons.

\section{MATERIALS AND METHODS}

NTS slices. The hindbrains of male Sprague Dawley rats (150-350 gm; Charles River Laboratories, Wilmington, MA) were prepared as described previously (Doyle and Andresen, 2001). All animal procedures were conducted with the approval of the University Animal Care and Use Committee in accordance with the U.S. Public Health Service Policy 
on Humane Care and Use of Laboratory Animals and the National Institutes of Health Guide for the Care and Use of Laboratory Animals. The hindbrain was removed and placed for $1 \mathrm{~min}$ in cold $\left(0-2^{\circ} \mathrm{C}\right)$ artificial CSF composed of (in mM): $125 \mathrm{NaCl}, 3 \mathrm{KCl}, 1.2 \mathrm{KH}_{2} \mathrm{PO}_{4}, 1.2$ $\mathrm{MgSO}_{4}, 25 \mathrm{NaHCO}_{3}, 10$ dextrose, and $2 \mathrm{CaCl}_{2}$ and bubbled with $95 \% \mathrm{O}_{2}$ and $5 \% \mathrm{CO}_{2}$. The medulla was trimmed to a $1 \mathrm{~cm}$ block (rostrocaudal) centered on the obex. A wedge of tissue was removed from the ventral surface to align the ST with the cutting plane when mounted in a vibrating microtome (VT-1000S; Leica, Nussloch, Germany). Slices (250 $\mu \mathrm{m}$ thick) cut with a sapphire knife (Delaware Diamond Knives, Wilmington, DE) contained the left ST in the same plane as the left NTS. Slices were submerged in a perfusion chamber and all recordings were performed at $34-37^{\circ} \mathrm{C}$ and a $\mathrm{pH}$ of 7.4 . Recording electrodes were filled with a solution composed of (in $\mathrm{mm}$ ): $10 \mathrm{NaCl}, 130 \mathrm{~K}$ gluconate, 11 EGTA, $1 \mathrm{CaCl}_{2}$, and $2 \mathrm{MgCl}_{2}$, 2 ATP, 0.2 GTP, 10 HEPES, pH 7.3, 295 mOsm. Neurons were recorded from the NTS within $200 \mu \mathrm{m}$ rostral or caudal from the obex and medial to the ST-medial NTS (mNTS). Patch electrodes, 1.8-3.5 $\mathrm{M} \Omega$, were guided to neurons using differential interference contrast (DIC) optics illuminated with infrared light (Axioskop; Zeiss, Oberkochen, Germany). Voltage-clamp recordings were made with an Axopatch 200A or Axoclamp 2A and pClamp 8 software (Axon Instruments, Foster City, CA). Only neurons with holding currents $\left(I_{\mathrm{h}}\right)$ of $\leq 100 \mathrm{pA}$ at $V_{\mathrm{H}}=-70 \mathrm{mV}$ for the $15 \mathrm{~min}$ control period (input resistance, $>150 \mathrm{M} \Omega$ ) were studied further. Synaptic currents were evoked with an ultrafine concentric bipolar stimulating electrode (200 $\mu \mathrm{m}$ outer diameter; Frederick Haer Co., Bowdoinham, ME) placed on the ST 1-3 $\mathrm{mm}$ from the recording electrode. Electrical stimuli were delivered from an isolated programmable stimulator (Master-8; A.M.P.I., Jerusalem, Israel) triggered to deliver a burst of stimuli (50$200 \mathrm{~Hz}$ ). Voltage-clamp protocols included $200 \mathrm{msec}$ voltage command steps ranging from -80 to $0 \mathrm{mV}$. All drugs were obtained from Tocris Cookson (Ballwin, MO). CAP and capsazepine were dissolved in either DMSO or ethanol at $1 \mu \mathrm{M}$ before diluting with external solution. DMSO or ethanol alone at the highest concentration in external solution had no effect on NTS neurons or synaptic transmission.

Aortic depressor nerve labeling. In 20-d-old rats anesthetized with pentobarbital $(50 \mathrm{mg} / \mathrm{kg})$, the aortic depressor nerve (ADN) was located and separated from the surrounding tissue $1 \mathrm{~cm}$ peripheral to joining the superior laryngeal nerve and entering the nodose ganglia. The lipophilic fluorescent dye 4-(4-dihexadecylamino)styryl)- $N$-methylpyridinium iodide (DiA; Molecular Probes, Eugene, OR) was placed on the ADN, fixed in place, and isolated by dental molding compound (Coltene, Mahwah, NJ) from adjacent nerves (Doyle and Andresen, 2001). Previous studies suggested that within several days dye begins to appear in the ST and NTS but that, over several months, the dye becomes concentrated over cell bodies within the NTS, with little in the distant ST axons (Mendelowitz et al., 1992). Thus, we allowed 2-3 months after surgery for dye transport to accumulate in the punctata in the mNTS; then horizontal brainstem slices were prepared (Doyle and Andresen, 2001). Fluorescent images of ADN contacts within the mNTS were captured by time integration for $>5 \mathrm{sec}$. The fluorescent images were acquired and digitally overlaid with real-time infrared DIC images from the Zeiss Axioskop at $40 \times$ objective using an Argus 20 image processor (Hammamatsu, Bridgewater, NJ) for anatomical neuron identification using aortic baroreceptor synaptic boutons as a marker (Balkowiec et al., 2000; Mendelowitz et al., 1992).

All data are presented as averages \pm SEM. Statistical comparisons were made using the unpaired Student's $t$ test, repeated measures (RM) ANOVA, one-way ANOVA, and Fisher's PLSD post hoc analysis when appropriate (see individual results) (Statview 4.57; Abacus Concepts, Calabasas, CA). $p<0.05$ indicated significant differences.

\section{RESULTS}

\section{Monosynaptic ST responses}

Cutting the brainstem slices horizontally preserved a lengthy segment of the ST in the same plane as the cell bodies of the NTS (Fig. 1A). This configuration allowed placement of the stimulating electrode on the visible ST at a sufficient distance from the recording area to eliminate focal activation of local interneurons and interconnecting fibers. Brief shocks (100 $\mu$ sec duration) passed through the stimulating electrode evoked EPSCs that were generally unitary, all-or-none responses with sharp stimulus current thresholds and little suprathreshold recruitment above
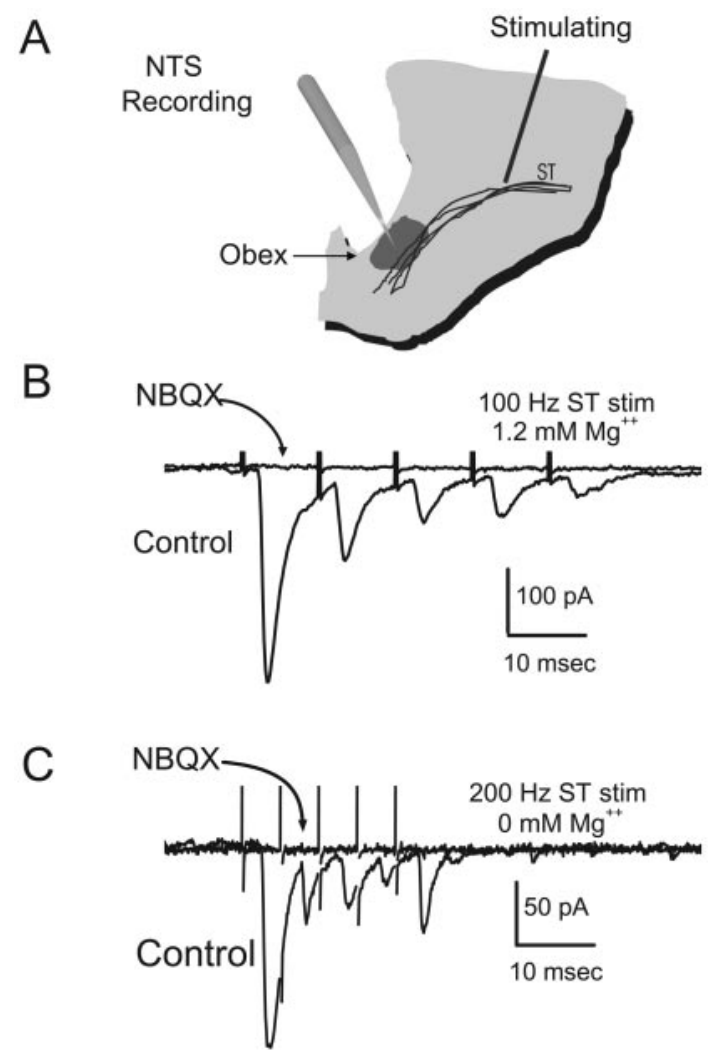

Figure 1. ST-evoked NTS synaptic responses in horizontal brainstem slices. $A$, Orientation of the rat brainstem slices in the horizontal plane allowed placement of the concentric bipolar stimulating electrode on the ST several millimeters from the recording region (dark gray) in the mNTS. $B$, ST activation evoked monosynaptic EPSCs in an mNTS neuron. $V_{\mathrm{M}}=$ $-70 \mathrm{mV}$. In the control, each ST shock (train of 5 pulses at $100 \mathrm{~Hz}$ ) evoked a short-latency EPSC with high reliability (latency, $1.9 \mathrm{msec}$; jitter, $38 \mu \mathrm{sec}$; no observed failures). The latency was averaged over 50 sweeps. Synaptic jitter was calculated as the SD of these trial latencies. Successive shocks evoked a frequency-dependent depression of EPSC amplitude. NBQX $(50 \mu \mathrm{M})$ completely blocked the EPSCs (flat trace). Note that this neuron possessed fluorescently labeled baroreceptor contacts and thus was anatomically monosynaptic to the ST (Fig. $7 A$, inset). $C$, In another neuron, ST evoked EPSCs with no NMDA current component in nominally $\mathrm{Mg}^{2+}$-free solution. NBQX completely blocked this highly reliable EPSC (latency, $2.3 \mathrm{msec}$; jitter, $46 \mu \mathrm{sec}$; no observed failures at $200 \mathrm{~Hz}$ ST stimulation).

threshold stimulus levels (Andresen and Yang, 1990). We identified responses as monosynaptic using electrophysiological criteria (Doyle and Andresen, 2001) as a combination of three measures of synaptic reliability: (1) absolute latency; (2) synaptic jitter (the $\mathrm{SD}$ of the intraneuronal latency); and (3) the absence of synaptic failures at high stimulus frequencies $(100-200 \mathrm{~Hz})$ to a burst of five shocks. We considered all synaptic responses with a jitter of $<150 \mu \mathrm{sec}$ as monosynaptic, a conservative criterion cutoff (Doyle and Andresen, 2001). Bursts of high-frequency stimuli consistently evoked EPSCs with minimal latency variation and very rare failures, despite rapid and substantial frequencydependent amplitude depression (Fig. 1B). Such EPSCs had short, nearly invariant latencies (averaging $4.28 \pm 0.21 \mathrm{msec}$ latency with a jitter of $84.0 \pm 3.7 \mu \mathrm{sec} ; n=72$ ) and few failures. The non-NMDA glutamate receptor antagonist NBQX blocked all such ST-evoked EPSCs (Fig. $1 B, C$ ), suggesting that these EPSCs are wholly mediated by non-NMDA glutamate receptors. At membrane potentials near rest, possible contributions to the 


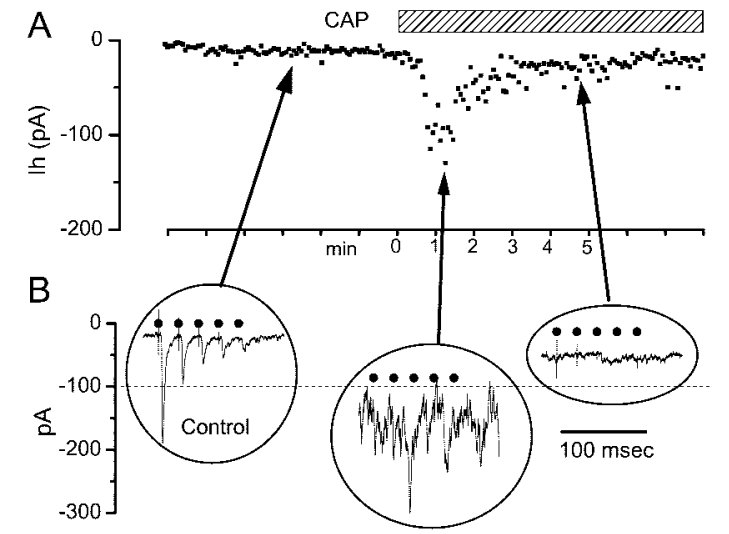

Figure 2. Responses of a representative CAP-sensitive mNTS neuron. $A$, $I_{\mathrm{h}}$ measured each $4 \mathrm{sec}$ at $-70 \mathrm{mV}$ were small and constant during the control period. Application of $100 \mathrm{~nm}$ CAP (bar) evoked an $I_{\text {cap }}$, which developed over $10-20 \mathrm{sec}$ and then subsided within $5 \mathrm{~min}$ in the continued presence of CAP. B, Bursts of ST stimulation evoked bursts of EPSCs in these neurons under control conditions; these evoked EPSCs were blocked by CAP. Expanded time segments from $A$ show the train of EPSCs evoked by five ST shocks (solid circles), and these were diminished at the peak of $I_{\text {cap }}$ (middle trace) when spontaneous synaptic activity increased. After $5 \mathrm{~min}$ in $100 \mathrm{~nm}$ CAP, $I_{\text {cap }}$ subsided and ST stimulation no longer evoked synaptic responses (bottom right trace). Synaptic responses in this cell met monosynaptic reliability criteria (latency, 3.12 msec; jitter, $120 \mu \mathrm{sec}$; no observable failures). The dotted line is a reference set at the approximate level of the peak $I_{\text {cap }}$.

synaptic current from NMDA receptors might be blocked by extracellular $\mathrm{Mg}^{2+}$ (Nowak et al., 1984). Nonetheless, NBQX fully blocked short-latency EPSCs $(n=19)$ even when recorded with $(n=11)$ or without $(n=8)$ extracellular $\mathrm{Mg}^{2+}$ (Fig. $\left.1 C\right)$ or at quite depolarized potentials (data not shown). Likewise, the NMDA receptor-specific antagonist AP-5 failed to alter such EPSCs ( $n=15$; data not shown) (Andresen and Yang, 1990).

\section{Vanilloid synaptic modulation}

Vanilloid receptors are expressed on a subset of somatic afferents, typically those with unmyelinated (C-type) and some with lightly myelinated (A $\delta$-type) axons (Stebbing et al., 1998; Szallasi and Blumberg, 1999; Caterina and Julius, 2001). In contrast, in a cranial afferent nerve trunk containing baroreceptor axons, CAP applied to the peripheral ADN trunk selectively blocked conduction of unmyelinated axons, whereas myelinated axons with A $\delta$ conduction velocities were not affected (Fan and Andresen, 1998). Because the central projections of cranial visceral afferents course in the ST, we hypothesized that VR1 on the central cranial afferent terminations corresponding to those peripheral C-type axons should modulate synaptic transmission presynaptically within the NTS.

Both CAP-sensitive and CAP-resistant synaptic responses were observed in neurons with EPSCs, meeting our reliability criteria for monosynaptic ST connections. In CAP-sensitive, secondorder NTS neurons, superfusion with 100 nM CAP had two transient effects (Fig. 2). CAP induced a rapidly developing inward current $\left(I_{\text {cap }}\right)$ (Fig. $\left.2 A\right)$. After $\sim 1 \mathrm{~min}, I_{\text {cap }}$ began to decline, despite sustained perfusion with CAP, and returned to near-control levels of the $I_{\mathrm{h}}$ within several minutes. Simultaneous with the increase in the $I_{\mathrm{h}}$, CAP evoked a large increase in spontaneous synaptic activity (Fig. $2 \mathrm{~B}$, middle). As $I_{\text {cap }}$ developed and spontaneous synaptic events increased, the amplitudes of ST-evoked EPSCs diminished. With a time course similar to the

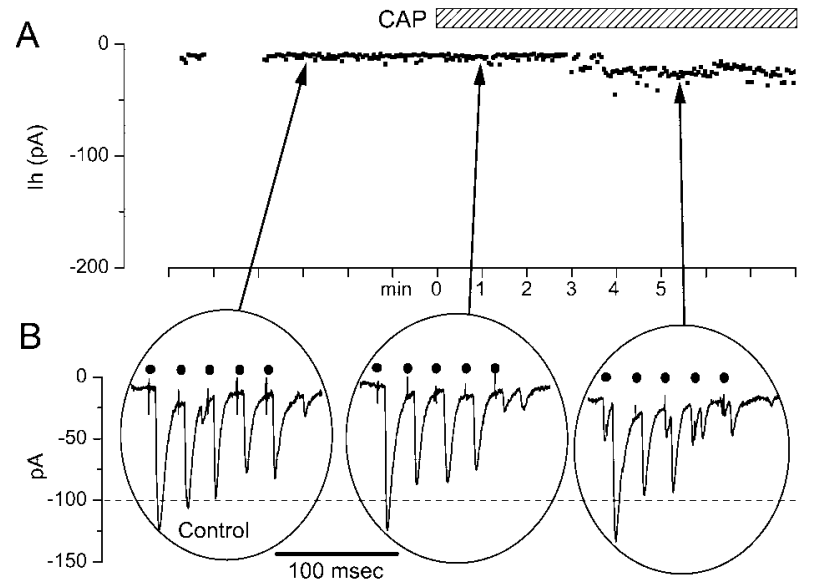

Figure 3. CAP had no effect on ST synaptic transmission in a CAPresistant mNTS neuron. $A$, ST evoked EPSCs in a CAP-resistant neuron. Synaptic responses in this cell met monosynaptic reliability criteria (latency, $4.7 \mathrm{msec}$; jitter, $107 \mu \mathrm{sec}$; no observable failures). Such responses showed typical amplitude depression to high-frequency trains of ST shocks. The gaps in data points during control are missing points. $B$, Bath application of CAP (100 nM) failed to alter the amplitude of the evoked EPSCs or to change the $I_{\mathrm{h}}$ (data not shown). The dotted line is a reference set at $100 \mathrm{pA}$.

decline of $I_{\text {cap }}$, the amplitude of ST-evoked EPSCs declined until they could no longer be detected (Fig. 2B, right). ST-evoked EPSCs remained blocked after $I_{\text {cap }}$ had subsided (Fig. 2B, right). Prolonged washing ( $>40 \mathrm{~min}$ ) gradually reversed the CAP blockade of ST-evoked synaptic transmission (see Fig. 9C). In CAPresistant NTS neurons (Fig. 3), CAP failed to alter synaptic transmission $(n=24)$. As with CAP-sensitive synaptic responses, CAP-resistant ST-evoked EPSCs were blocked by NBQX $(n=6$; results not shown) and met the reliability criteria for monosynaptic connections.

CAP-sensitive neurons, on average, had significantly longer latencies than CAP-resistant neurons $(p=0.011 ; 4.65 \pm 0.27$ msec, $n=48$, vs $3.53 \pm 0.28 \mathrm{msec}, n=24$, respectively). However, the distributions of latency values were indistinct, and the ranges of latencies substantially overlapped between the two groups (Fig. 4). Despite the differences in latency, the synaptic jitters of the EPSCs were similar for the two groups $(p=0.72 ; 85.9 \pm 7.3$ vs $83.1 \pm 4.3 \mu \mathrm{sec}$; CAP-resistant vs CAP-sensitive). The pairing of CAP sensitivity with longer EPSC latency may partially reflect differences in the known peripheral conduction velocities of cranial afferents in which $\mathrm{C}$-types are CAP-sensitive and $\mathrm{A} \delta$-types are CAP-resistant (Fan and Andresen, 1998).

The average $I_{\text {cap }}(108.1 \pm 22.9 \mathrm{pA} ; n=21)$ during CAP peaked at $3 \mathrm{~min}$; by $7 \mathrm{~min}$ it declined to $\sim 35 \%$ of that peak in the continued presence of CAP (Fig. 5A). During CAP, the STevoked EPSC amplitude declined steadily to near zero by $6 \mathrm{~min}$ on average (Fig. 5B). Application of the competitive VR1 receptor antagonist capsazepine produced a concentration-dependent reduction in $I_{\text {cap }}(p=0.013$; RM ANOVA) (Fig. 6A) and reduced the inhibition of ST-evoked EPSC amplitude by CAP ( $p=0.0007$; RM ANOVA) (Fig. 6B). Capsazepine alone had no effect on $I_{\mathrm{h}}$ or EPSC amplitudes $(p>0.7)$. These results are consistent with CAP acting through VR1 receptors.

\section{Identified baroreceptor endings}

Many modalities of cranial visceral afferents enter the CNS at the NTS (Andresen and Kunze, 1994). In the medial area of the 


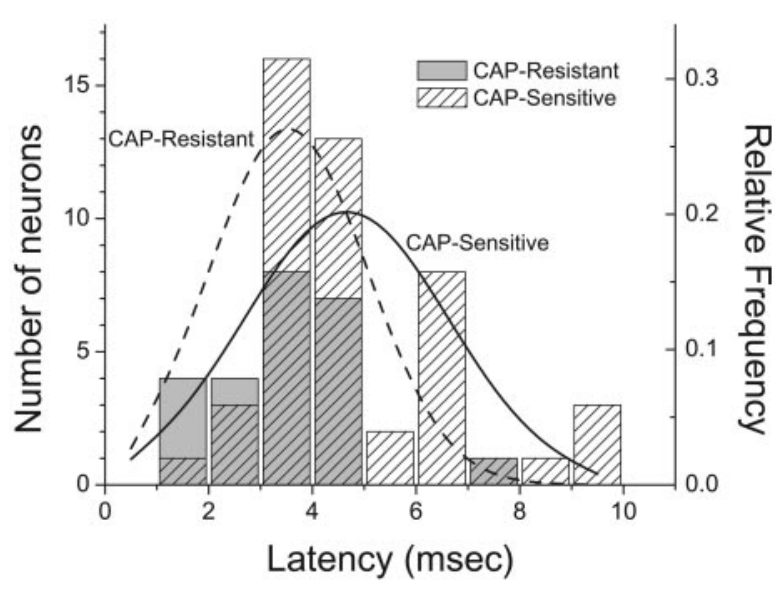

Figure 4. Histograms plot the distribution of latency values for CAPsensitive (solid line) and CAP-resistant (dashed line) EPSCs of mNTS neurons. The mean latency for CAP-sensitive neurons was significantly longer than for CAP-resistant neurons (unpaired $t$ test; $p=0.011 ; 4.65 \pm$ $0.27 \mathrm{msec}, n=48$ vs $3.53 \pm 0.28 \mathrm{msec}, n=24$, respectively). Solid lines (CAP-sensitive) and dashed lines (CAP-resistant) represent normal distributions.

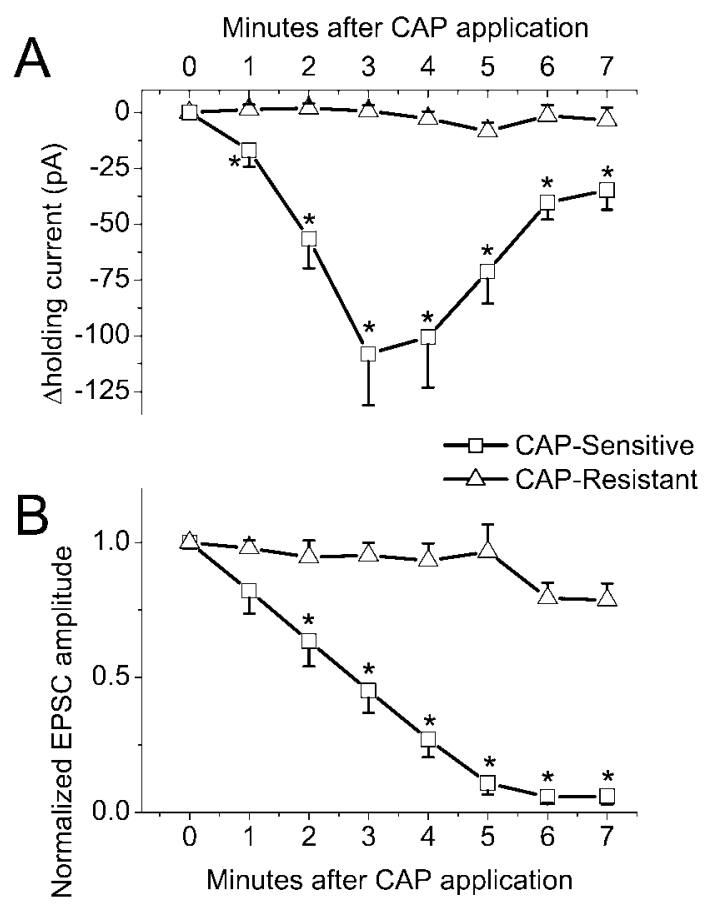

Figure 5. Average time course of the changes in $I_{\mathrm{h}}$ and amplitude of ST-evoked EPSCs in CAP-sensitive and CAP-resistant second-order neurons. $A$, CAP application significantly increased the $I_{\mathrm{h}}$ in CAP-sensitive compared with CAP-resistant neurons $(n=21$ and 19, respectively; $p<$ 0.0001; RM ANOVA; error bars indicate SEM). Individual time point comparisons were made with one-way ANOVA $\left({ }^{*} p \leq 0.0174\right)$. B, CAP inhibited the EPSC amplitude significantly in CAP-sensitive compared with CAP-resistant neurons ( $n=21$ and 19 , respectively; $p<0.0001 ; \mathrm{RM}$ ANOVA; error bars indicate SEM). Individual time point comparisons were made with one-way ANOVA $\left({ }^{*} p \leq 0.0222\right)$.

dorsal caudal NTS, neurons receive input from aortic baroreceptors that travel centrally from the ADN (Sapru et al., 1981). Up to $90 \%$ of these ADN baroreceptor axons are unmyelinated (Andresen et al., 1978). Such C-fiber neurons have conduction velocities, ion channels, and receptors similar to spinal nociceptive
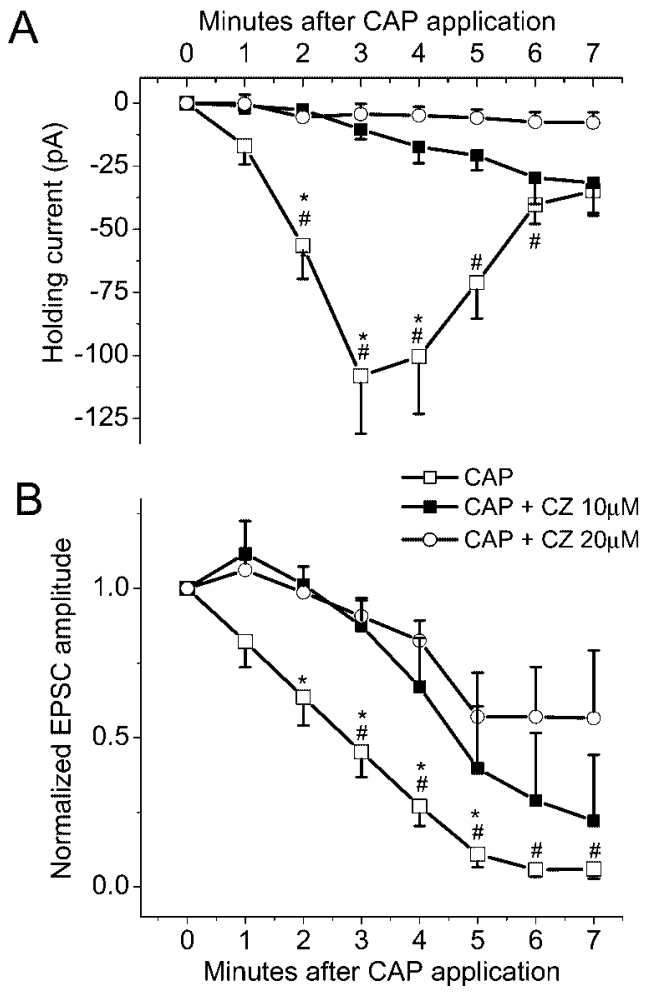

Figure 6. The VR1 antagonist capsazepine $(C Z)$ inhibited the effects of CAP. All neurons were CAP-sensitive. $A$, Both 10 and $20 \mu \mathrm{M} \mathrm{CZ}$ significantly (each $n=4$ ) reduced $I_{\mathrm{h}}$ changes in response to $100 \mathrm{nM}$ CAP $(n=21 ; p=0.0131$; RM ANOVA). Individual time point comparisons of CAP alone, CAP plus $10 \mu \mathrm{M}$ capsazepine, and CAP plus $20 \mu \mathrm{M}$ capsazepine by one-way ANOVA with Fisher's PLSD post hoc analysis are shown $\left({ }^{*} p<0.05\right.$ between CAP alone and CAP plus $10 \mu \mathrm{M}$ capsazepine; ${ }^{\#} p<0.05$ between CAP alone and CAP plus $20 \mu \mathrm{M}$ capsazepine; error bars indicate SEM). $B$, Both 10 and $20 \mu \mathrm{M}$ capsazepine inhibited and delayed the reduction of the evoked EPSC amplitude ( $p=0.0007$; RM ANOVA). Individual time point comparisons of EPSC in CAP alone, CAP plus $10 \mu \mathrm{M}$ capsazepine, and CAP plus $20 \mu \mathrm{M}$ capsazepine by one-way ANOVA with Fisher's PLSD post hoc analysis are shown $\left({ }^{*} p<\right.$ 0.05 between CAP alone and CAP plus $10 \mu \mathrm{M}$ capsazepine; ${ }^{\#} p<0.05$ between CAP alone and CAP plus $20 \mu \mathrm{M}$ capsazepine; error bars indicate SEM).

sensory neurons (Lawson, 1992). As with somatic nociceptors (Holzer, 1991; Caterina et al., 1997; Helliwell et al., 1998), CAP depolarizes ADN peripheral endings consistent with the presence of VR1 on peripheral axons. To identify baroreceptor synaptic contacts within the NTS, and thus locate second-order neurons, we placed the lipophilic dye DiA on cervical portions of the ADN to anterogradely label central terminations (Mendelowitz et al., 1992). The presence of these fluorescent baroreceptor boutons on the cell bodies of NTS neurons identified them anatomically as second-order (see Figs. 7, 9). Patch pipettes were directed under infrared DIC microscopy to record from these NTS neurons (Fig. 7, inset) (Doyle and Andresen, 2001). ST activation evoked very-short-latency EPSCs with minimal jitter and no failures in most of the identified neurons even at highfrequency stimulation (Fig. $1 B$ ). In these identified baroreflex neurons, CAP induced a transient $I_{\text {cap }}$ comparable with other NTS neurons receiving electrophysiologically defined monosynaptic EPSCs (Fig. 7). Similar results were found in four additional DiA-labeled NTS neurons. 


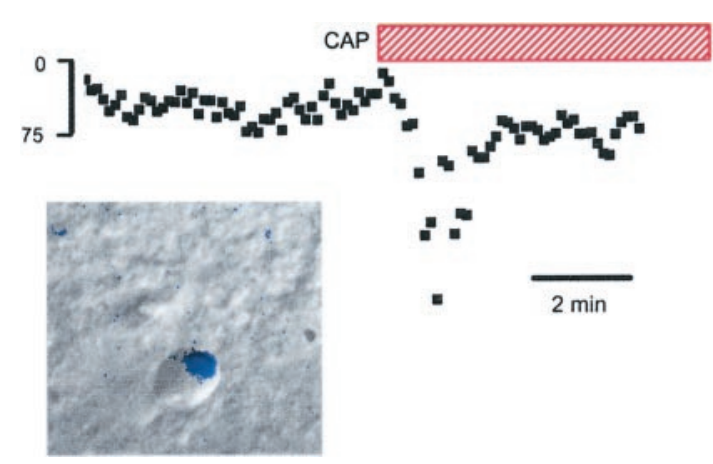

Figure 7. CAP response recorded in an mNTS neuron with an anatomically identified sensory input. The DiA fluorescent image of ADN terminals (inset, blue) was superimposed on the infrared DIC image of this mNTS neuron. Fluorescent DiA labeling of aortic baroreceptor afferents was found primarily on cell bodies and indicated directly that these neurons received visceral sensory contacts. The outline of the neuron in the DIC focal plane is apparent with the associated fluorescently labeled contacts. ST stimulation-evoked EPSCs met monosynaptic latency criteria. CAP (100 nM, red bar) evoked a transient $I_{\text {cap }}$ similar to unlabeled NTS neurons. $I_{\text {cap }}$ peaked in 1-2 min and subsided to control levels within $5 \mathrm{~min}$ in the continued presence of CAP.
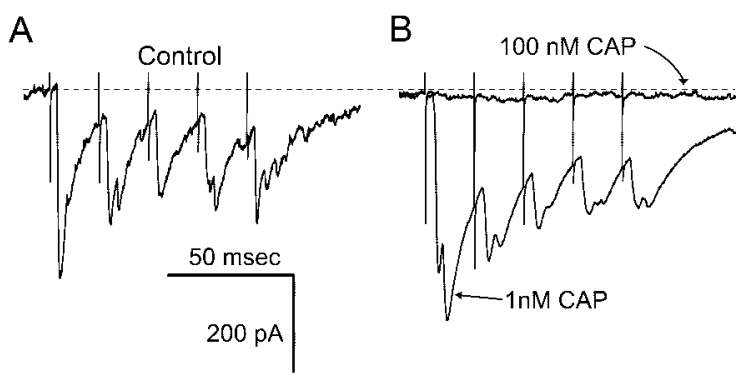

Figure 8. Low concentrations of CAP enhanced ST-evoked EPSCs. $A$, In control solutions, ST stimulation evoked a train of EPSCs. $B$, After 3 min in $1 \mathrm{~nm}$ CAP, identical trains of ST stimuli evoked larger EPSCs, with no change in the $I_{\mathrm{h}}$. Higher concentrations of CAP $(100 \mathrm{nM})$ blocked the EPSCs ( flat trace). The dotted line indicates the control level of the $I_{\mathrm{h}}$. All traces are the means of five consecutive responses to ST stimulus trains containing bursts of five shocks.

\section{Presynaptic actions of CAP}

The effects of CAP in mNTS neurons are consistent with a presynaptic action on cranial visceral afferents of the ST. The VR1 ion channel complex fluxes cations, including calcium (Tominaga et al., 1998). Low concentrations of CAP (1 nM) facilitated synaptic transmission and increased the size of the evoked EPSC (Fig. 8) without changing the $I_{\mathrm{h}}$. Prolonged perfusion with higher concentrations of CAP (100 nM) abolished ST-evoked EPSCs as in all CAP-sensitive neurons. Such results are consistent with a presynaptic CAP action increasing the probability of transmitter release.

If CAP is acting at presynaptic sensory terminals within the mNTS or the ST, the postsynaptic effects of CAP are most likely mediated by released glutamate-activating non-NMDA receptors. To test this, we pretreated neurons with the non-NMDA antagonist NBQX. Such pretreatment blocked ST-evoked EPSCs and completely prevented the development of $I_{\text {cap }}$ both in unlabeled monosynaptic neurons $(n=7)$ and in an identified baroreceptor neuron (Fig. 9A) $(n=1)$. Thus, presumably because presynaptic VR1 is still activated by CAP, the glutamate released does not act on additional postsynaptic glutamate receptor sub-

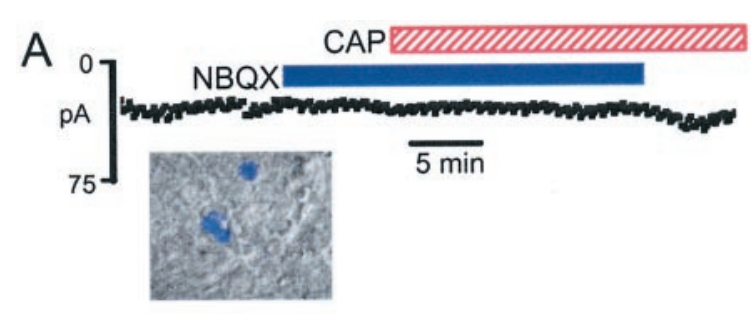

B
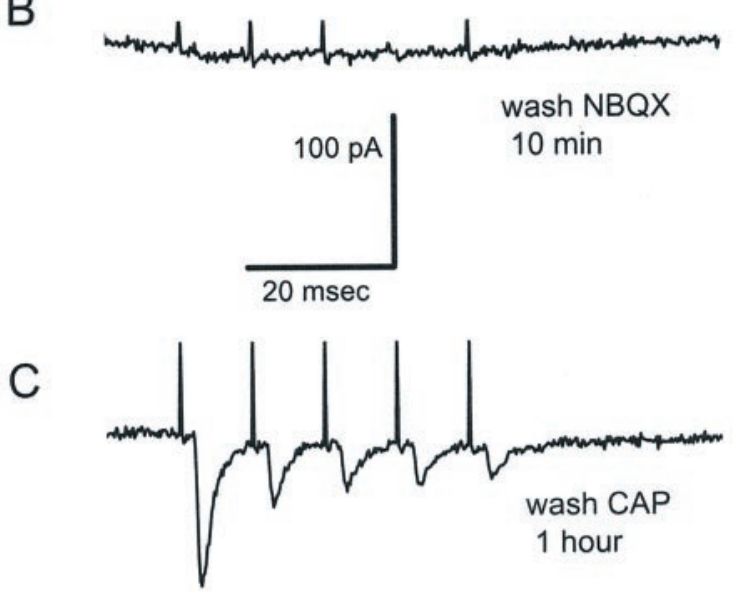

Figure 9. $A$, The non-NMDA antagonist NBQX $(50 \mu \mathrm{M})$ prevented $I_{\text {cap }}$ in a baroreceptor-labeled mNTS neuron (inset). ST-evoked EPSCs in this neuron met the reliability criteria for monosynaptic responses (see control response traces for this neuron in Fig. $1 B$ ). NBQX blocked all ST-evoked synaptic activity and prevented $I_{\text {cap }}$ in response to 100 nM CAP. $B$, CAP continued to block ST-evoked EPSCs after the removal of NBQX, demonstrating CAP sensitivity in this neuron. $C$, Evoked EPSCs returned after the removal of CAP, indicating intact postsynaptic receptors.

types (e.g., NMDA receptors). To check for CAP sensitivity in these neurons, NBQX was removed during CAP exposure. STevoked EPSCs remained blocked in CAP in the absence of NBQX (Fig. 9B) and were considered CAP-sensitive. Recovery of the ST-evoked EPSC by perfusion with control solution required up to $1 \mathrm{hr}$ (Fig. 9C). The recovery of the evoked EPSCs demonstrates the reversible nature of CAP effects. These data also suggest that postsynaptic responses to ST activation by either electrical stimulation or CAP application in identified secondorder NTS neurons are mediated by glutamate via non-NMDA receptors. The similarity between labeled and unlabeled neurons suggests that this is likely a general property of cranial visceral afferents.

Spontaneous synaptic activity was prominent in many secondorder mNTS neurons and was evident as EPSCs occurring out of synchrony with ST electrical shocks (Fig. 10, left, arrow). The application of CAP blocked ST-triggered EPSCs after 3-5 min of exposure in all CAP-sensitive neurons. However, substantial spontaneous synaptic activity remained, despite the effective blockade of ST-evoked events by CAP (Fig. 10, middle). The application of NBQX blocked this spontaneous synaptic activity during CAP (Fig. 10, right). Thus, non-NMDA receptors are required for postsynaptic CAP actions, and CAP does not compromise the non-NMDA receptor function itself. To further test this, we applied glutamate agonists to a CAP-sensitive neuron after $I_{\text {cap }}$ had subsided and in the continuing presence of CAP and the addition of TTX (Fig. 11). In the presence of CAP and TTX, the application of glutamate but not NMDA induced a 


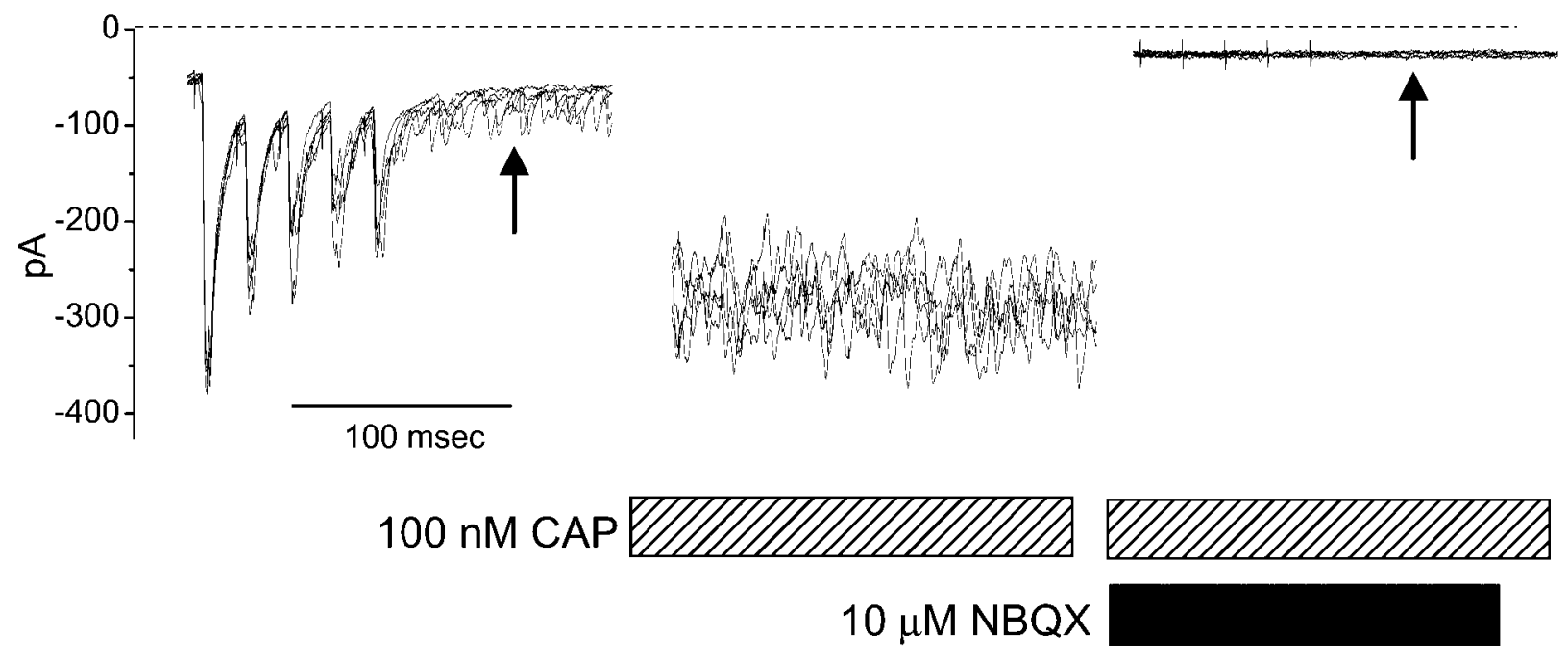

Figure 10. Non-NMDA receptors were functional during CAP. Spontaneous EPSCs occurred unsynchronized to ST electrical stimulation before CAP application (left, arrow), and this activity was relatively high in this neuron. During $100 \mathrm{~nm}$ CAP application, the $I_{\mathrm{h}}\left(V_{\mathrm{H}}=-70 \mathrm{mV}\right)$ increased, as did the frequency of spontaneous EPSCs (middle, hatched bar). ST-triggered EPSCs were blocked by CAP. Application of NBQX (10 $\mu \mathrm{M})$ during $I_{\text {cap }}$ eliminated changes in $I_{\mathrm{h}}$ and blocked spontaneous synaptic activity (right, arrow). These results are consistent with dependence on non-NMDA receptors for ST EPSCs, spontaneous EPSCs, and $I_{\text {cap }}$. Because spontaneous EPSCs persisted in CAP, they appear to arise from CAP-resistant sources (A-type or nonafferent contacts). Each panel displays five consecutive, superimposed sweeps during $50 \mathrm{~Hz}$ of ST stimulation. Each train was separated by 2 sec. Dotted line indicates the zero $I_{\mathrm{h}}$ level for reference.

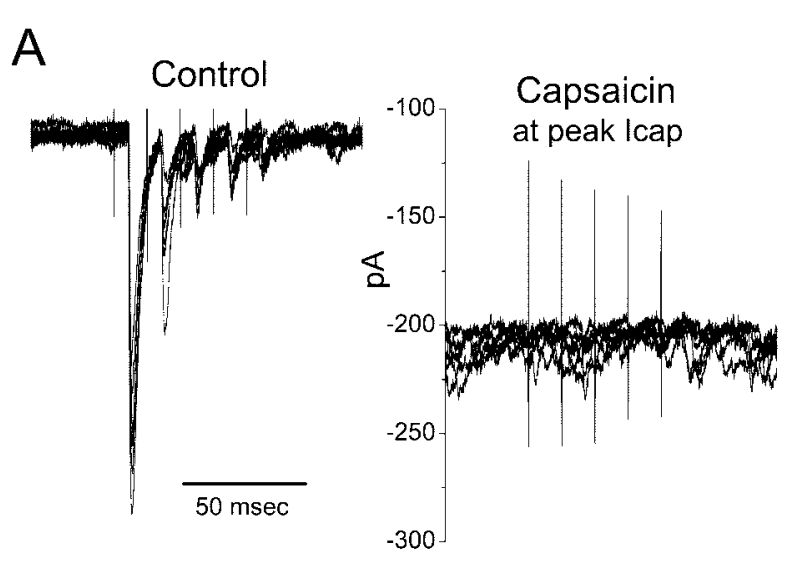

B

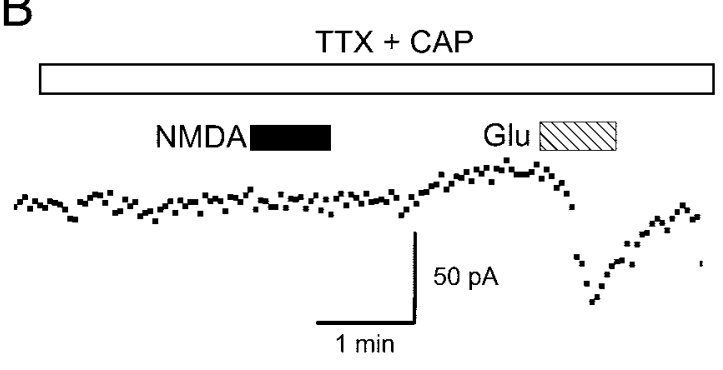

Figure 11. Glutamate $(G l u)$ receptors were functional during CAP treatment. $A$, A $100 \mathrm{~nm}$ concentration of CAP increased the spontaneous synaptic activity (note the spontaneous synaptic currents in the initial segment of overlaid traces) and blocked ST-evoked monosynaptic EPSCs (latency, $6.12 \mathrm{msec}$; jitter, $82 \mu \mathrm{sec}$ ). $B$, After $I_{\text {cap }}$ subsided and in the presence of CAP and TTX, a brief application of NMDA (2 mM) had no effect on the $I_{\mathrm{h}}$, but a brief application of glutamate $(1 \mathrm{~mm})$ rapidly induced a large $I_{\text {cap }}$ consistent with intact non-NMDA receptors. substantial $I_{\text {cap }}($ Fig. $11 B)$, verifying that non-NMDA receptors were functionally intact during prolonged CAP treatment.

\section{DISCUSSION}

\section{VR1 facilitates glutamate release in the NTS}

Our studies suggest that the presence of VR1 on presynaptic terminals of the ST is a functional marker for two very different pools of second-order mNTS neurons. The activation of cranial visceral afferent axons within the ST evokes short-latency EPSCs that are mediated by non-NMDA receptors. Responses to bursts of afferent ST shocks yield large-amplitude EPSCs that depress markedly in a frequency-dependent manner; this pattern, together with the somatic location of afferent boutons, appears to be characteristic of this NTS afferent synapse (Andresen et al., 2001) as well as other brainstem sensory synapses (Trussell, 1999). In the present studies, we found two classes of secondorder mNTS neurons, CAP-sensitive and CAP-resistant. We did not observe intermediate responses (partial EPSC sensitivity to CAP) and conclude that functionally there appears to be little overlap in these two afferent classes (i.e., a mixture of VR1 and non-VR1 afferents) impinging on any single mNTS neuron. This segregation of inputs in the NTS is distinctly different from the observations of dorsal horn second-order neurons, at which mixed CAP-sensitive and CAP-resistant inputs were common (Nakatsuka et al., 2002).

CAP actions on sensitive NTS neurons required the presence of available non-NMDA receptors, because the selective antagonist NBQX blocked both CAP $I_{\text {cap }}$ and the CAP increase in spontaneous synaptic inputs. Reports about the spinal cord suggest a substantial participation of NMDA receptors in CAPinitiated responses (Nagy et al., 1993). Although CAP has been suggested to have potential nonspecific actions (Szallasi and Blumberg, 1999), the absence of effects in CAP-resistant secondorder mNTS neurons and the effectiveness of NBQX blockade in CAP-sensitive neurons indicate that CAP is highly specific in the nanomolar range of concentrations used. Exogenous glutamate 
agonist responses were intact in the presence of CAP at times when ST synaptic transmission was blocked. This appears to rule out a direct postsynaptic CAP action that might compromise non-NMDA receptor function. Thus, our results are consistent with a presynaptic action activating specific afferent terminals at VR1 by CAP. With prolonged exposure, CAP blocks ST-evoked EPSCs. NMDA receptors were not activated by ST stimulation or by CAP even in the absence of extracellular $\mathrm{Mg}^{2+}$. These findings reinforce the suggestion that glutamate released from cranial afferent processes does not have access to postsynaptic NMDA receptors (Andresen and Yang, 1990). In the spinal cord, NMDA receptor participation is often associated with polysynaptic pathways (Headley and Grillner, 1990); as in other areas of the CNS, the NMDA receptor can contribute to synaptic plasticity such as "wind up" in spinal cord neurons (Nagy et al., 1993). Microinjection of NMDA or NMDA receptor antagonists into medial portions of the NTS strongly affects resting blood pressure and cardiovascular reflex responses (Kubo and Kihara, 1988; Ohta and Talman, 1994), indicating important functional contributions of NMDA receptors. Our results indicate that such NMDA receptors must be located primarily at higher-order neurons, and that perhaps the capacity for glutamatergic plasticity may reside at these locations. The present results in cranial visceral pathways raise the question of whether similar cellular mechanistic distinctions might exist in visceral afferent pathways in the spinal cord.

The precise mechanism(s) by which prolonged CAP produces blockade of electrically evoked ST sensory transmission is not clear. CAP might either block afferent axonal conduction as it does peripherally or inactivate the presynaptic voltage-gated ion channels required for neurotransmitter release through sustained depolarization. Alternatively and/or in addition, CAP might deplete glutamate-containing vesicles by persistent ligand binding and activation of the terminals via VR1. Either mechanism could account for the disappearance of $I_{\text {cap }}$ during sustained CAP exposure and the prolonged time course for the recovery of ST-evoked EPSCs after the removal of CAP. These observations support CAP actions limited to presynaptic sensory afferent sites and are consistent with VR1 localization at cranial visceral sensory presynaptic endings similar to spinal nociceptors. Our studies focused on the medial portions of NTS; CAP may have additional effects in regions that receive different sensory afferents.

\section{Differential A- and C-fiber afferent processing pathways}

Our results demonstrate two pharmacologically distinct ST pathways to mNTS neurons, CAP-sensitive and CAP-resistant. This dichotomy parallels the pharmacological sensitivity of a cranial sensory nerve trunk, the ADN, in which CAP blocks the conduction of recorded volleys from $\mathrm{C}$ but not $\mathrm{A} \delta$ axons (Fan and Andresen, 1998). The cardiovascular reflex responses from this CAP-sensitive component of the ADN were identical to those remaining when an anodal current selectively blocked A-fiber conduction (i.e., equivalent to C-type activation) (Fan et al., 1999). If our conclusion that CAP-resistant afferents are A-type and CAP-sensitive are C-type is accurate, then these pathways appear to separate at the second-order neuron in the NTS, because CAP responses were all or none. This segregation of afferent inputs to different populations of second-order mNTS neurons, if found to be generalized across other regions of the NTS, could represent a very different organization than that within many spinal sensory pathways, in which overlap or conver- gence of A- and C-type and CAP-sensitive and CAP-resistant afferents is prominent (Nakatsuka et al., 2002).

Evidence for the convergence of afferent inputs of different modalities onto single NTS neurons is varied. Electrical activation of multiple cranial visceral afferent nerves in anesthetized cats suggested that convergence onto single NTS neurons was relatively rare (249 of 292) using the carotid sinus, aortic and vagal nerves alone or in combination (Donoghue et al., 1985). Likewise, few NTS neurons in the cat were activated by both myelinated and unmyelinated aortic-nerve afferents, suggesting that convergence across these axon subtypes was infrequent (Donoghue et al., 1981). However, results are often difficult to generalize across species and techniques. In the mouse and in the rat, mechanical or chemical activation of visceral afferents indicated that multiple inputs are common in some single NTS neurons but not in others and can influence neurons over many seconds (Felder and Mifflin, 1994; Mifflin, 1996; Paton, 1998; SilvaCarvalho et al., 1998; Paton et al., 1999). However, the precise timing of inputs is often lost in such manipulations and, although potentially more physiologic, it is difficult to distinguish monosynaptic from polysynaptic pathways. Given the loosely viscerotopic organization of the NTS (Loewy, 1990), it is unclear whether the different results represent differences in regional organization across the NTS. Very few studies have been conducted that discriminate A- from C-type cranial visceral afferent inputs to the NTS. Thus, the failure in our present studies to observe the convergence of both A- and C-type afferents on single mNTS neurons will require considerable additional investigation to determine how general this pattern might be with regard to all of the NTS.

From the perspective of NTS and brainstem processing, our results in the mNTS are potentially of interest within the context of the organization of cardiovascular reflexes. The two classes of ST afferent inputs corresponding to arterial baroreceptors may contribute to the mechanisms by which A- and C-fiber reflex pathways operate. Studies selectively activating these afferent subclasses demonstrate distinct frequency-dependent differentiation of CAP-sensitive and CAP-resistant ADN axons (Fan and Andresen, 1998). Furthermore, the reflex pathways that regulate heart rate integrate $\mathrm{A}$ - and $\mathrm{C}$-fiber inputs differently from the pathways for blood pressure control (Fan et al., 1999). Very little is known about the CNS mechanisms that are responsible for such differences. Thus, our present results on ST-NTS synaptic transmission indicate that CAP can be used to separate the synaptic responses of $\mathrm{A}$ - and $\mathrm{C}$-fiber pathways at their first synapses. This approach may be useful in determining the cellular basis for the differential afferent processing along these reflex pathways. Initial results suggest that the second-order NTS neurons themselves express classes of potassium channels differentially, and that these critically contribute to frequency-dependent encoding (Bailey et al., 2002). Given that the cardiac baroreflex pathway can consist of as few as two brainstem neurons (e.g., NTS to nucleus ambiguus) before contacting the peripheral parasympathetic postganglionic neurons at the heart (Loewy and Spyer, 1990), substantial functional differentiation may occur as early as the ST afferent synapse in the NTS.

\section{REFERENCES}

Andresen MC, Kunze DL (1994) Nucleus tractus solitarius: gateway to neural circulatory control. Annu Rev Physiol 56:93-116.

Andresen MC, Yang M (1990) Non-NMDA receptors mediate sensory afferent synaptic transmission in medial nucleus tractus solitarius. Am J Physiol 259:H1307-H1311. 
Andresen MC, Krauhs JM, Brown AM (1978) Relationship of aortic wall baroreceptor properties during development in normotensive and spontaneously hypertensive rats. Circ Res 43:728-738.

Andresen MC, Doyle MW, Jin Y-H, Bailey TW (2001) Cellular mechanisms of baroreceptor integration at the nucleus tractus solitarius. Ann NY Acad Sci 940:132-141.

Bailey TW, Jin Y-H, Doyle MW, Andresen MC (2002) Vanilloidsensitive afferents activate neurons with prominent A-type potassium currents in nucleus tractus solitarius. J Neurosci 22:8230-8237.

Balkowiec A, Kunze DL, Katz DM (2000) Brain-derived neurotrophic factor acutely inhibits AMPA-mediated currents in developing sensory relay neurons. J Neurosci 20:1904-1911.

Burnstock G (2000) P2X receptors in sensory neurones. Br J Anaesth $84: 476-488$.

Caterina MJ, Julius D (2001) The vanilloid receptor: a molecular gateway to the pain pathway. Annu Rev Neurosci 24:487-517.

Caterina MJ, Schumacher MA, Tominaga M, Rosen TA, Levine JD, Julius D (1997) The capsaicin receptor: a heat-activated ion channel in the pain pathway. Nature 389:816-824.

Donoghue S, Fox RE, Kidd C, McWilliam PN (1981) The terminations and secondary projections of myelinated and non-myelinated fibres of the aortic nerve in the cat. Q J Exp Physiol 66:405-422.

Donoghue S, Felder RB, Gilbey MP, Jordan D, Spyer KM (1985) Postsynaptic activity evoked in the nucleus tractus solitarius by carotid sinus and aortic nerve afferents in the cat. J Physiol (Lond) 360:261-273.

Doyle MW, Andresen MC (2001) Reliability of monosynaptic transmission in brain stem neurons in vitro. J Neurophysiol 85:2213-2223.

Fan W, Andresen MC (1998) Differential frequency-dependent reflex integration of myelinated and nonmyelinated rat aortic baroreceptors. Am J Physiol 275:H632-H640.

Fan W, Schild JH, Andresen MC (1999) Graded and dynamic reflex summation of myelinated and unmyelinated rat aortic baroreceptors. Am J Physiol 277:R748-R756.

Felder RB, Mifflin SW (1994) Baroreceptor and chemoreceptor afferent processing in the solitary tract nucleus. In: Nucleus of the solitary tract (Barraco RA, ed), pp 169-186. Boca Raton, FL: CRC.

Guo A, Vulchanova L, Wang J, Li X, Elde R (1999) Immunocytochemical localization of the vanilloid receptor 1 (VR1): relationship to neuropeptides, the $\mathrm{P}_{2} \mathrm{X}_{3}$ purinoceptor, and IB4 binding sites. Eur J Neurosci 11:946-958.

Headley PM, Grillner S (1990) Excitatory amino acids and synaptic transmission: the evidence for a physiological function. Trends Pharmacol Sci 11:205-211.

Helliwell RJA, McLatchie LM, Clarke M, Winter J, Bevan S, McIntyre P (1998) Capsaicin sensitivity is associated with the expression of the vanilloid (capsaicin) receptor (VR1) mRNA in adult rat sensory ganglia. Neurosci Lett 250:177-180.

Holzer P (1991) Capsaicin: cellular targets, mechanisms of action, and selectivity for thin sensory neurons. Pharmacol Rev 43:143-201.

Kubo T, Kihara M (1988) Evidence of $N$-methyl-D-aspartate receptormediated modulation of the aortic baroreceptor reflex in the rat nucleus tractus solitarius. Neurosci Lett 87:69-74.

Lawson SN (1992) Morphological and biochemical cell types of sensory neurons. In: Sensory neurons: diversity, development, and plasticity (Scott SA, ed), pp 27-59. New York: Oxford UP.

Loewy AD (1990) Central autonomic pathways. In: Central regulation of autonomic functions (Loewy AD, Spyer KM, eds), pp 88-103. New York: Oxford UP.
Loewy AD, Spyer KM (1990) Vagal preganglionic neurons. In: Central regulation of autonomic functions (Loewy AD, Spyer KM, eds), pp 68-87. New York: Oxford UP.

Mendelowitz D, Yang M, Andresen MC, Kunze DL (1992) Localization and retention in vitro of fluorescently labeled aortic baroreceptor terminals on neurons from the nucleus tractus solitarius. Brain Res 581:339-343.

Mezey E, Toth ZE, Cortright DN, Arzubi MK, Krause JE, Elde R, Guo A, Blumberg PM, Szallasi A (2000) Distribution of mRNA for vanilloid receptor subtype 1 (VR1), and VR1-like immunoreactivity, in the central nervous system of the rat and human. Proc Natl Acad Sci USA 97:3655-3660.

Mifflin SW (1996) Convergent carotid sinus nerve and superior laryngeal nerve afferent inputs to neurons in the NTS. Am J Physiol 271:R870-R880.

Miles R (1986) Frequency dependence of synaptic transmission in nucleus of the solitary tract in vitro. J Neurophysiol 55:1076-1090.

Nagy I, Maggi CA, Dray A, Woolf CJ, Urban L (1993) The role of neurokinin and $N$-methyl-D-aspartate receptors in synaptic transmission from capsaicin-sensitive primary afferents in the rat spinal cord in vitro. Neuroscience 52:1029-1037.

Nakatsuka T, Furue H, Yoshimura M, Gu JG (2002) Activation of central terminal vanilloid receptor-1 receptors and $\alpha \beta$-methylene-ATPsensitive $\mathrm{P} 2 \mathrm{X}$ receptors reveals a converged synaptic activity onto the deep dorsal horn neurons of the spinal cord. J Neurosci 22:1228-1237.

Nowak L, Bregestovski P, Ascher P, Herbet A, Prochiantz A (1984) Magnesium gates glutamate-activated channels in mouse central neurones. Nature 307:462-465.

Ohta H, Talman WT (1994) Both NMDA and non-NMDA receptors in the NTS participate in the baroreceptor reflex in rats. Am J Physiol 267:R1065-R1070.

Paton JFR (1998) Pattern of cardiorespiratory afferent convergence to solitary tract neurons driven by pulmonary vagal $\mathrm{C}$-fiber stimulation in the mouse. J Neurophysiol 79:2365-2373.

Paton JF, Li YW, Kasparov S (1999) Reflex response and convergence of pharyngoesophageal and peripheral chemoreceptors in the nucleus of the solitary tract. Neuroscience 93:143-154.

Saper CB (2000) Pain as a visceral sensation. Prog Brain Res 122:237-243.

Sapru HN, Gonzalez E, Krieger AJ (1981) Aortic nerve stimulation in the rat: cardiovascular and respiratory responses. Brain Res Bull 6:393-398.

Silva-Carvalho L, Paton JFR, Rocha I, Goldsmith GE, Spyer KM (1998) Convergence properties of solitary tract neurons responsive to cardiac receptor stimulation in the anesthetized cat. J Neurophysiol 79:2374-2382.

Stebbing MJ, McLachlan EM, Sah P (1998) Are there functional P2X receptors on cell bodies in intact dorsal root ganglia of rats? Neuroscience 86:1235-1244.

Szallasi A, Blumberg PM (1999) Vanilloid (capsaicin) receptors and mechanisms. Pharmacol Rev 51:159-212.

Tominaga M, Caterina MJ, Malmberg AB, Rosen TA, Gilbert H, Skinner K, Raumann BE, Basbaum AI, Julius D (1998) The cloned capsaicin receptor integrates multiple pain-producing stimuli. Neuron 21:531-543.

Trussell LO (1999) Synaptic mechanisms for coding timing in auditory neurons. Annu Rev Physiol 61:477-496. 